\title{
Guest Editorial: Special Issue on Deep Learning with Small Samples
}

\author{
A B S T R AC T
}

It was a big challenge to learn a deep neural network with excellent generalizability from only a small number of training samples. Recently, deep learning has achieved promising performance for certain small-sample problems, for example, by transferring the "knowledge" learned from other datasets containing rich labeled data or generating synthetic samples. However, many challenging topics remain with small-sample deep leaning techniques, such as data augmentation, discriminative feature learning, prior construction and meta-learning. Therefore, the goal of this special issue is to collect the latest developments in various aspects of deep learning with small samples. After at least two rounds of review, we select 12 articles from nearly 40 submissions into this special issue; these articles can be roughly grouped into three categories: literature review (1), methodological work for some generic tasks (2-6) and for some specific applications and data (7-12). We hope that these selected articles could promote many more excellent researches in smallsample deep learning.

1. A concise review of recent few-shot meta-learning methods: Li et al. provide a concise review on recent representative methods in few-shot meta-learning, which are categorised into four branches according to their technical characteristics, as well as some challenges and future prospects in few-shot meta-learning.

2. Domain generalization via optimal transport with metric similarity learning: Zhou et al. aim to improve the domain generalization, i.e. to learn from multiple source domains and generalize to a target domain with unknown statistics, by constraining the label similarity when extracting the invariant features and exploiting the class-specific cohesion and separation of features across domains. They adopt an optimal transport with the Wasserstein distance to constrain the label similarity and a metric learning objective to achieve a distinguishable classification boundary.

3. Adding geodesic information and stochastic patch-wise image prediction for small dataset learning: For image segmentation and prediction, Hammoumi et al. propose an image-augmentation method, by adding structural information with specific distance transform to the input image data, and a patch-based procedure with a stratified sampling method for inference, to handle the case with limited number of training samples.

4. Deep InterBoost networks for small-sample image classification: Li et al. propose a simple AdaBoost-like ensemble deep network called InterBoost for small-

$\mathrm{ORCID}(\mathrm{s})$ sample image classification. In the training phase, InterBoost first randomly generates two sets of complementary weights for the training data to separately train two base networks of the same structure, and then updates these weights through interaction between the two trained base networks.

5. Deep time series models for scarce data: After identifying two types of scarce data in classification or regression of multivariate time series, Wang et al. propose a model called sparse functional multilayer perceptron to handle the sparsity in the time-series covariates. Mathematical arguments are provided to investigate the effectiveness of the proposed method.

6. Deep video action clustering via spatio-temporal feature learning: Peng et al. propose a deep network of small-sample video action clustering, to learn the similarity among unlabeled video samples and then cluster them. The proposed method develops a 3D U-Net self-representation generator, to learn spatio-temporal features and subspace structures of video clips for the similarity graph construction and clustering.

7. Sketch-specific data augmentation for freehand sketch recognition: To address the problems of limited training data and substantial intra-class variance of freehand sketches, Zheng et al. propose a sketch-specific data-augmentation approach. They introduce a Bezier pivot based deformation strategy to enrich the training data and use a mean stroke reconstruction method to generate a set of novel types of sketches with smaller intra-class variance.

8. Few-shot palmprint recognition based on similarity metric hashing network: Liu et al. propose a similarity metric hashing network (SMHNet) for few-shot palmprint recognition. SMHNet involves a structural similarity (SSIM) index block to extract the features of palmprint images at the structural level, as well as a hashing block to encode the features into hashing codes for the convenience of large-scale feature storage and retrieval.

9. An intelligent fault diagnosis model based on deep neural network for few-shot fault diagnosis: Wang and $\mathrm{Xu}$ develop an intelligent fault diagnosis model for few-shot fault diagnosis using similarities of sample pairs to classify samples. The proposed model contains a twin-network module for learning features and a metric-learning module for predicting similarities of sample pairs. The proposed model is evaluated on two cases of bearing fault diagnosis.

10. The diagnosis of inter-turn short circuit of permanent magnet synchronous motor based on deep learning 
and small fault samples: Li et al. propose a method to detect the inter-turn short circuit problem for permanent magnet synchronous motors, by adapting a conditional generative adversarial net to expand few fault samples and an optimised sparse autoencoder to enhance the generalization ability of the network.

11. Improving speech recognition models with small samples for air traffic control systems: To tackle the problem of small samples in training a practical automatic speech recognition model for air traffic control systems, Lin et al. propose a method leveraging both unsupervised pretraining to learn speech representations from unlabeled samples and transfer learning to finetune baseline models with a joint corpus consisting of baseline samples and new transcribed target samples.

12. HAL: Hybrid active learning for efficient labeling in medical domain: To address the challenge of constructing large datasets of labeled medical images, Wu et al. propose a hybrid active learning (HAL) framework for efficient labeling by integrating active learning into deep learning. The proposed HAL exploits a hybrid sampling strategy considering both sample diversity and prediction loss.

To conclude this editorial, the guest editors would like to thank all the authors and reviewers for their great contributions and the editorial office and the Editors-in-Chief for their consistent support.

\section{Declaration of Conflicts of Interest}

The authors declare no conflicts of interest.

Jing-Hao Xue

University College London, UK

Jufeng Yang

Nankai University, China

Xiaoxu Li

Lanzhou University of Technology, China

Yan Yan

Xiamen University, China

Yujiu Yang

Tsinghua University, China

Zongqing Lu

Tsinghua University, China

Zhanyu Ma

Beijing University of Posts and Telecommunications, China 